\title{
Theranostics with radiation-induced ultrasound emission (TRUE)
}

\author{
Elijah Robertson and Liangzhong Xiang* \\ School of Electrical and Computer Engineering \\ Institute for Biomedical Engineering, Science and Technology \\ University of Oklahoma, Norman, OK 73019, USA \\ Stephenson Cancer Center at the University of Oklahoma \\ Norman, OK 73019, USA \\ *xianglzh@ou.edu
}

Received 14 January 2018

Accepted 18 March 2018

Published 9 April 2018

\begin{abstract}
Two novel ultrasound imaging techniques with imaging contrast mechanisms are in the works: X-ray-induced acoustic computed tomography (XACT), and nanoscale photoacoustic tomography (nPAT). XACT has incredible potential in: (1) biomedical imaging, through which a 3D image can be generated using only a single X-ray projection, and (2) radiation dosimetry. nPAT as a new alternative of super-resolution microscopy can break through the optical diffraction limit and is capable of exploring sub-cellular structures without reliance on fluorescence labeling. We expect these new imaging techniques to find widespread applications in both pre-clinical and clinical biomedical research.
\end{abstract}

Keywords: X-ray-induced acoustic computed tomography (XACT); low-dose CT; threedimensional (3D) volumetric imaging; nanoscale photoacoustic tomography; label-free superresolution.

\section{Introduction}

Since the founding of the practice of medicine, humans have sought to understand the intricate details of the functions of the living body. Before the 20th century, when technology was lacking, the primary means to obtain such information was through surgery, a highly invasive and potentially dangerous solution. In the last 100 years, however, immense progress has been made in the development of new imaging techniques by which doctors can noninvasively observe various structures ranging from internal organs down to microscopic entities. ${ }^{1}$ While these techniques have been extremely useful, new biomedical imaging technologies are essential to fundamentally improve the detection, treatment, and prevention of disease. ${ }^{2}$ This paper

${ }^{*}$ Corresponding author.

This is an Open Access article published by World Scientific Publishing Company. It is distributed under the terms of the Creative Commons Attribution 4.0 (CC-BY) License. Further distribution of this work is permitted, provided the original work is properly cited. 
describes the exciting work the theranostics with radiation-induced ultrasound emission (TRUE) lab at The University of Oklahoma has performed on two novel imaging techniques.

\section{X-Ray-Induced Acoustic Computed Tomography (XACT)}

Since Röntgen discovered X-rays more than 100 years ago, X-ray imaging has been an invaluable tool in medical diagnosis, biology and materials science. $^{3-5}$ X-ray computed tomography (CT) has proved tremendously useful for noninvasive medical imaging ever since its inception nearly 50 years ago. However, one issue that is intrinsic to CT is its large radiation dosage. It is estimated that up to $2 \%$ of cancer cases are the result of the radiation obtained from CT imaging. ${ }^{6}$ This risk therefore can potentially negate many of the benefits provided by CT. An answer to this unsolved problem is in the works at the TRUE lab, X-ray-induced acoustic computed tomography (XACT). XACT utilizes a newly discovered physics principle, that X-rays can generate acoustic waves within tissue. ${ }^{7}$ While CT relies upon a rotating X-ray source and many X-ray projections to obtain a three-dimensional (3D) image, XACT can generate a 3D image through one single $\mathrm{X}$-ray projection, drastically decreasing radiation dose. While XACT potentially has many desirable features, this revolutionary fact alone has given enough warrant to pursue this new technique.

The physics behind XACT is simple: the sample to be imaged is irradiated with pulsed X-rays. Innershell electrons then absorb these X-ray photons and become excited, causing photoelectrons to be released. This transfer of energy increases the temperature and, thus, produces atomic vibrations. The ultimate result is the emission of ultrasound waves in all directions. ${ }^{7}$ As these waves are $3 \mathrm{D}$ in nature, a single projection allows for $3 \mathrm{D}$ reconstruction via the induced acoustic waves (Fig. 1). The amplitude of the acoustic wave is proportional to X-ray absorption; therefore, the contrast mechanism for this technique is the same as that of $\mathrm{CT}$ in its ability to differentiate tissue. ${ }^{7}$

$\mathrm{XACT}$ imaging as a novel biomedical imaging modality was first proposed and demonstrated in $2013,{ }^{7}$ and has since been studied by different groups all over the world in various pre-clinical applications. ${ }^{8-11}$ XACT has been tested using a

(a)

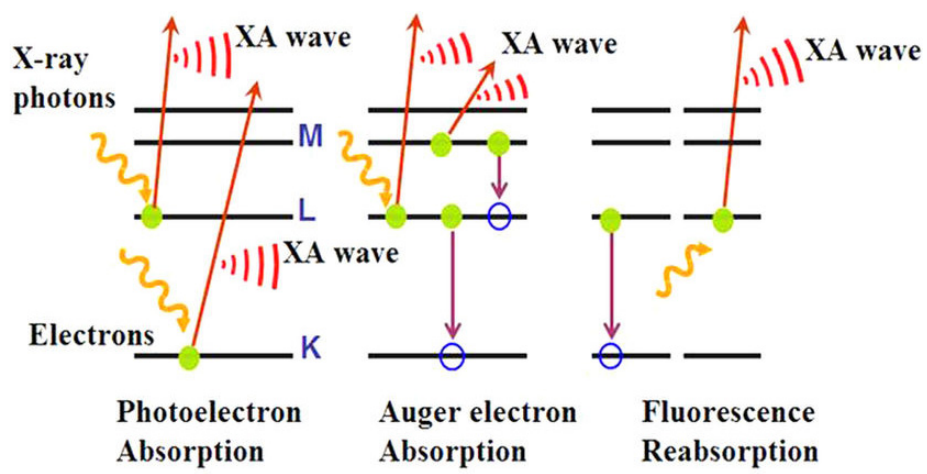

(b)
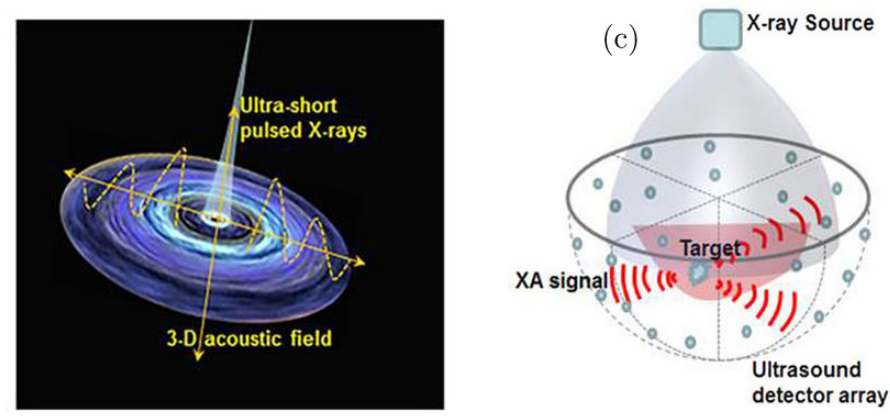

Fig. 1. (a) Schematic of the main processes which contribute to XA signals. M, L and K represent the electron shells around an atom's nucleus. (b) Schematic of the 3D acoustic field generated by ultrashort-pulsed X-rays. (c) Schematic diagram of 3D XACT. (Fig. 1 of Ref. 12). 
variety of materials including water, lead, bone, etc. in both imaging and dosimetry applications. Single ultrasound transducers with low center frequencies have been used for X-ray-induced acoustic signal collection in XACT imaging investigations. A typical XACT imaging system with a single ultrasound transducer requires mechanical scanning for acquisition of a two-dimensional (2D) image, leading to hours of scanning and requiring multiple X-ray pulses. ${ }^{7,8,12}$ Recently, a new XACT imaging system that offers rapid and high resolution 2D images has been developed and tested (Fig. 2). ${ }^{13}$ In this new system, a sample is irradiated by a nanosecondpulsed X-ray source, giving off acoustic waves in all directions. Instead of using a single transducer detector, a ring array of piezoelectric ultrasound transducers detects the acoustic waves, and converts them to electrical signals. The resulting signals are then processed using a back-projection algorithm, yielding a reconstructed image. It should be noted that as this current system uses a ring-array of transducers, only a single 2D slice of the sample can be obtained. When a spherical or cup-shaped array is used, 3D imaging would be possible. However, due to equipment limitations, only $2 \mathrm{D}$ slices have been obtained.

A couple of experiments using XACT imaging systems are highlighted here. About $150 \mu \mathrm{m}$ thick gold fiducial markers were tested and were shown to be in good agreement with the corresponding CT image. This was done using a single transducer which was mechanically scanned around the sample. Spatial resolution was determined to be $350 \mu \mathrm{m} .{ }^{12}$ More recently, using a ring array of higher center-frequency transducers, lead sheets with thickness of $150 \mu \mathrm{m}$ were shaped into the OU logo and then imaged with the fast XACT imaging system. This experiment resulted in a spatial resolution of $138 \mu \mathrm{m} .^{13}$

A few challenges presented with these experiments were primarily due to equipment limitations. Both of these images required a large number of $\mathrm{X}$-ray pulses to obtain a decent signal-to-noise ratio (SNR). This large number of pulses can be reduced by two methods, both of which, at the time of writing, are being investigated. The first is to

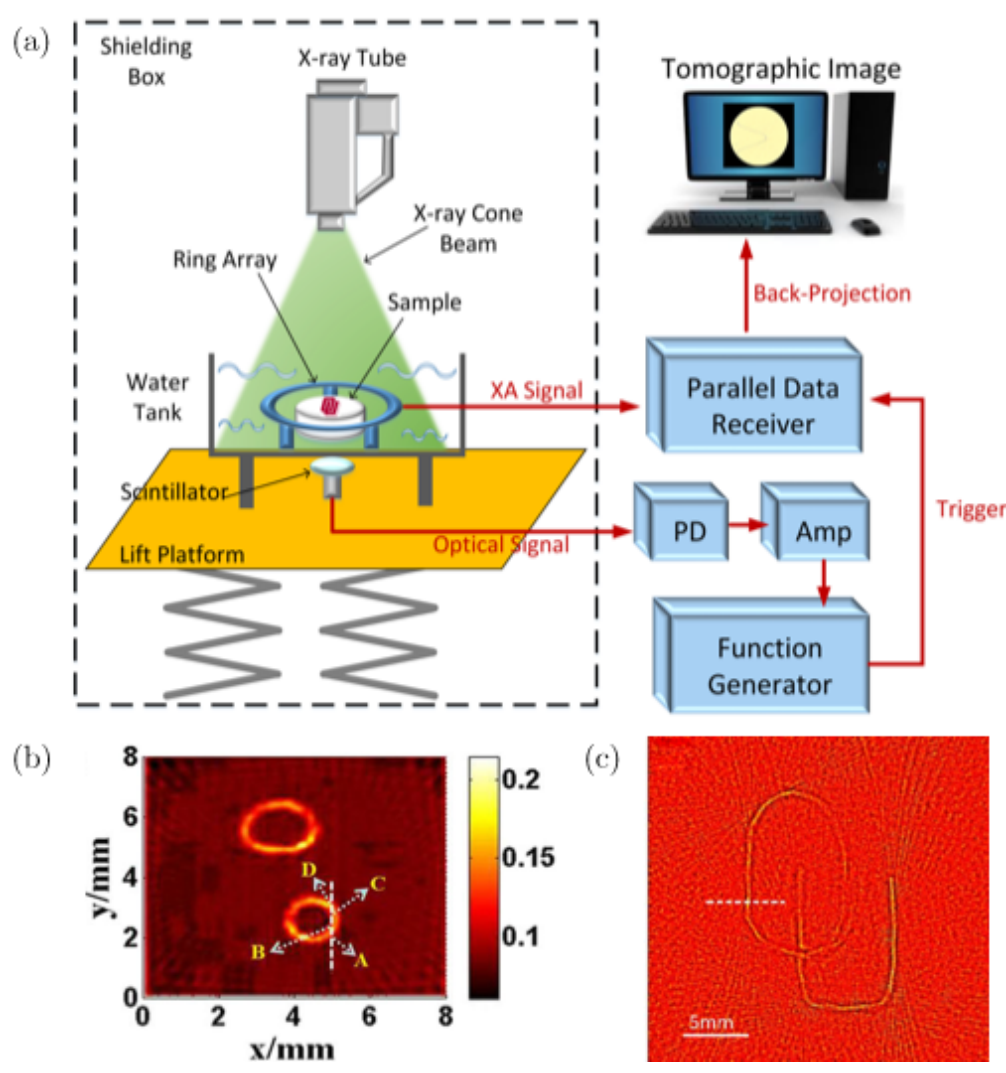

Fig. 2. (a) Schematic diagram of XACT imaging system. A scintillator/photodiode combination is activated by the X-rays and is used to tell the data receiver when to start collecting signals from the ultrasound transducer array (Fig. 1 of Ref. 13). (b) Gold fiducial marker XACT image (Fig. 3 of Ref. 12). (c) Lead OU logo XACT image (Fig. 3 of Ref. 13). 
increase the amplification of each transducer in the ring array, as they are currently amplified at only $52 \mathrm{~dB}$. An amplifier with enough channels to match each transducer element on the ring-array is necessary to do this. Second, the fluence of the X-ray tube can be increased. The X-ray source has a large, unfocused field-of-view, which provides smaller amplitude of the acoustic waves than those produced by focused X-rays. ${ }^{13}$

Considering the use of XACT imaging in breast imaging, minimal X-ray exposure can generate a 3D acoustic image of the breast, which dramatically reduces the radiation dose of patients when compared to conventional breast CT. ${ }^{12}$ Simulation studies have shown that XACT can image microcalcifications within breast tissue with doses as low as $0.4 \mathrm{mGy}, 24$ times smaller than that of dedicated breast $\mathrm{CT} .{ }^{14}$ Bone mineral density mapping is also possible with XACT. ${ }^{13}$

$\mathrm{XACT}$ imaging also has been proposed to be used for a variety of radiation dosimetry applications during radiation therapy (Fig. 3). ${ }^{7,15}$ XACT has numerous advantageous characteristics that make it a promising technique for water tank dosimetry applications. First, there is a linear relationship between deposited dose and induced pressure in a homogeneous medium. Additionally, $\mathrm{XACT}$ is dependent on the dose deposited per pulse, meaning it can be considered energy and dose rate independent. ${ }^{8}$ Also, XACT does not perturb the radiation beam provided the transducers are placed outside the beam path. These features of XACT simplify calibration and eliminate the need for many of the corrective factors required by other dosimetry techniques. After the initial demonstration of XACT, ${ }^{7}$ various groups have worked on applying XACT to radiotherapy dosimetry applications in two main areas: relative water tank dosimetry and in vivo dosimetry. These studies demonstrated the feasibility of using XACT as a dosimetry technique in a clinical radiotherapy environment.

The use of XACT in either diagnostic imaging or dosimetry is dependent on the accessibility of shortduration pulsed X-ray sources, ${ }^{13}$ which are not widely available in clinical applications. To be considered for widespread use, a cost-effective method to provide easy access to such sources is necessary. Special training will also be required to work the equipment and interpret resulting images.
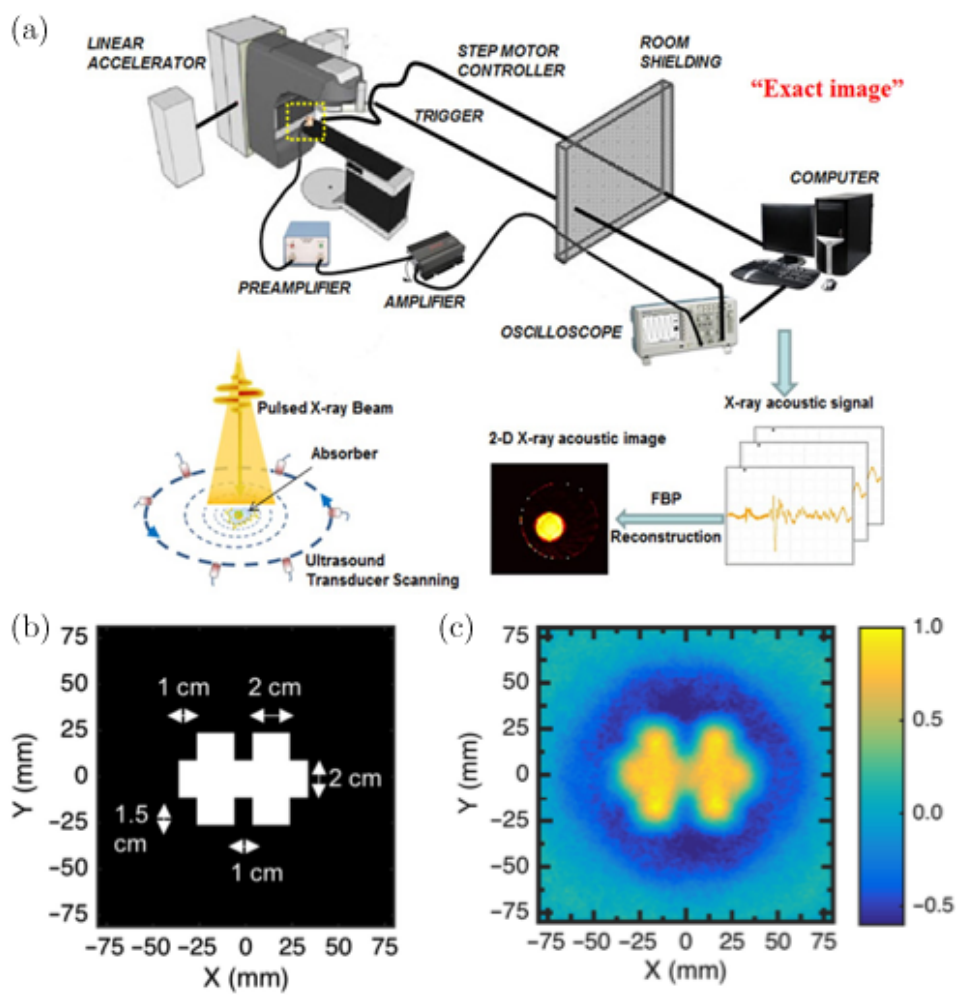

Fig. 3. (a) Schematic diagram of XACT imaging system using a linear accelerator (Fig. 2 of Ref. 7). (b) Linac irradiated field dimensions. (c) Resulting XACT image produced by irradiated field in (b) (Fig. 11 of Ref. 8). 
If these are addressed, XACT has much potential to transform modern clinical care.

\section{Nanoscale Photoacoustic Tomography (nPAT)}

In the late 19th century, it was found that optical microscopes could not achieve resolution past 250 $\mathrm{nm}$. This limit has long prevented researchers from imaging on the nanometer level until recently, when many new super-resolution techniques have emerged. ${ }^{16}$ One of the most recent techniques, fluorescent super-resolution microscopy, won the 2014 Nobel Prize in Chemistry by breaking the diffraction limit and allowing for observation of many biological structures indeterminable by conventional fluorescence microscopy. ${ }^{17-19}$ This and other prominent techniques unfortunately require external labeling with photoswitchable fluorophores, which can require extensive preparation and are often difficult to use. ${ }^{20,21}$ They can also perturb the observed structure itself. $^{22}$ Label-free super-resolution imaging, thus, would provide immense advances in the super-resolution fields.
Here, we are developing a novel super-resolution microscopy method to explore subcellular structures without relying on florescence tagging via the photoacoustic effect. In the photoacoustic phenomenon, light is absorbed by molecules and converted to heat. The subsequent thermoelastic expansion generates an acoustic wave, which is detected by acoustic detectors to form images. ${ }^{23-29}$ In nanoscale photoacoustic tomography (nPAT), an ultra-short $(<\mathrm{ps})$ pulsed laser is used for the generation of $\mathrm{GHz}$ ultrasound waves. $^{30}$ The ultrahigh frequency acoustic waves are detected by a pump-probe technique to achieve nanoscale tomographic imaging, whereby the contrast is dependent on differing optical absorptions between tissue for particular wavelengths ${ }^{31,32}$; the high resolution, however, does come at a cost to imaging depth, which is relatively low. One example of applying nPAT imaging is to monitor malarial parasite invasion of human erythrocytes in real time at the nanometer scale to understand malaria disease progression (Fig. 4). ${ }^{33}$ The advances of the nPAT imaging technique not only will benefit the biomedical imaging sciences, but also provide fundamental understanding of

(a)

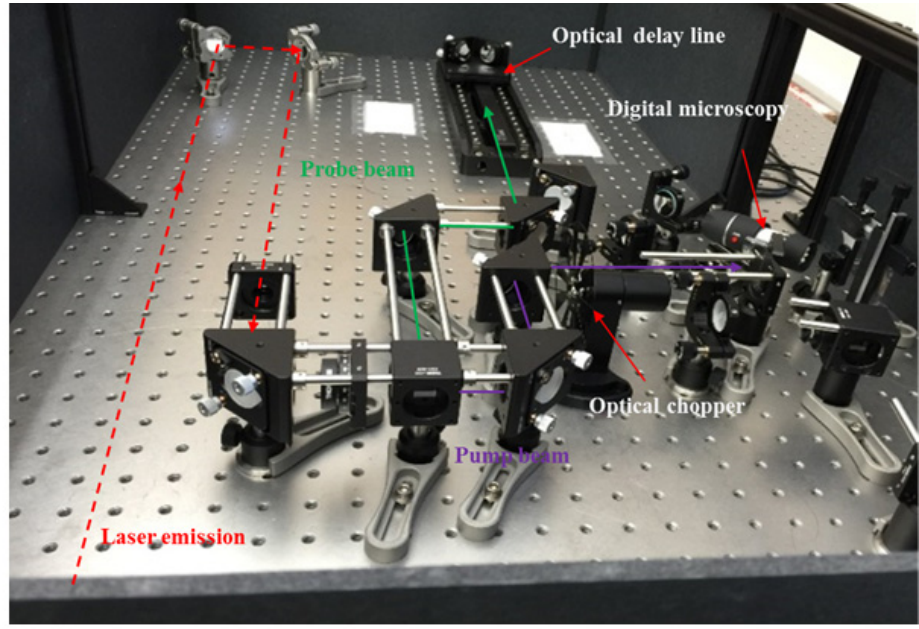

(b)

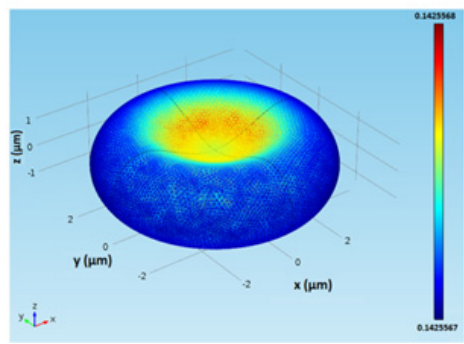

(c)

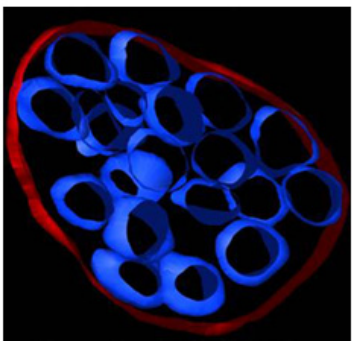

Fig. 4. (a) nPAT System setup. Pump-probe technique is used to provide ultra-high frequency detection. (b) Spatial distribution of temperature rise on a single regular biconcave red blood cell (Fig. 6 of Ref. 32). (c) 3D nPAT imaging reconstruction of malarial parasite invasion (in blue) of human erythrocytes (in red), where the biconcave shape is no longer present. 
biological processes (hemoglobin in red blood cells, cytochromes in mitochondria, and DNA/RNA within the cell nucleus) and medicine.

\section{Summary}

We believe that XACT and nPAT will find broad applications in both basic research and clinical care. The aforementioned predictions represent the authors' biased opinions of the most promising prospects of XACT in both diagnostic imaging for either quantitative bone density mapping or breast cancer, and in vivo radiation dosimetry during radiation therapy. nPAT also, as a label-free imaging technique, has much potential to revolutionize the super-resolution imaging fields, providing new ways to monitor diseases and understand biological processes. Undoubtedly, many more applications for either of these techniques will be identified. We look forward to seeing the impact of TRUE technology on biomedicine.

\section{Conflicts of Interest}

The authors have no relevant conflicts of interest to disclose.

\section{Acknowledgments}

The authors gratefully acknowledge PHF Team Science Grant Program and Diabetes CoBRE Pilot Project from The University of Oklahoma Health Sciences Center, and Seed grant from the College of Engineering at The University of Oklahoma for funding. The authors would like to thank the lab members Shanshan Tang, Ali Zarafshani, Jian Chen, Pratik Samant, Rowzat Faiz, Siqi Wang, and the under-graduate research assistants for their contributions in the TRUE lab.

Research is supported by PHF Team Science Grant Program and Diabetes CoBRE Pilot Project from The University of Oklahoma Health Sciences Center, and Seed grant from the College of Engineering at The University of Oklahoma.

\section{References}

1. J. C. Gore, "Biomedical imaging: Current and future trends," Institue of Physics, (2015) http:// medicalphysicsweb.org/cws/article/opinion/60791.
2. R. I. Pettigrew, "Director's Corner," National Institute of Biomedical Imaging and Bioengineering, (2017) https://www.nibib.nih.gov/about-nibib/directorspage.

3. W. L. Chao, B. D. Harteneck, J. A. Liddle, E. H. Anderson, D. T. Attwood, "Soft X-ray microscopy at a spatial resolution better than $15 \mathrm{~nm}, "$ Nature 435, 1210-1213 (2005).

4. E. de Smit et al., "Nanoscale chemical imaging of a working catalyst by scanning transmission X-ray microscopy," Nature 456, 222-225 (2008).

5. L. Kipp et al., "Sharper images by focusing soft X-rays with photon sieves," Nature 414, 184-188 (2001).

6. D. J. Brenner, E. J. Hall, "Computed tomography - An increasing source of radiation exposure," N. Engl. J. Med. 357, 2277-2284 (2007).

7. L. Xiang, B. Han, C. Carpenter, G. Pratx, Y. Kuang, L. Xing, "X-ray acoustic computed tomography with pulsed X-ray beam from a medical linear accelerator," Med. Phys. 40, 010701 (2013).

8. S. Hickling et al., "Experimental evaluation of X-ray acoustic computed tomography for radiotherapy dosimetry applications," Med. Phys. 44(2), 608-617 (2017).

9. S. H. M. Hickling, I. El Naqa, "Feasibility of X-ray acoustic computed tomography as a tool for noninvasive volumetric in vivo dosimetry," Int. J. Radiat. Oncol. 90, S843 (2014).

10. D. R. T. Sampaio, J. H. Uliana, A. A. O. Carneiro, J. F. Pavoni, T. Z. Pavan and L. F. Borges, "X-ray acoustic imaging for external beam radiation therapy dosimetry using a commercial ultrasound scanner," in 2015 IEEE International Ultrasonics Symposium (IUS) (Taipei, 2015), pp. 1-4.

11. J. Kim, E.-Y. Park, Y. Jung, B. C. Kim, J. H. Kim, C.-Y. Yi, I. J. Kim, C. Kim, "X-ray acoustic-based dosimetry using a focused ultrasound transducer and a medical linear accelerator," IEEE Trans. Radiat. Plasma Med. Sci. 1(6), 534-540 (2017).

12. L. Xiang et al., "High resolution X-ray-induced acoustic tomography," Sci. Rep. 6, 26118 (2016).

13. S. Tang et al., "X-ray-induced acoustic computed tomography with an ultrasound transducer ringarray," Appl. Phys. Lett. 110(10), 103504, 2017.

14. S. Tang, K. Yang, Y. Chen, L. Xiang, "X-ray induced acoustic computed tomography for 3D breast imaging: A simulation study," Med. Phys. (2018).

15. L. Xiang, B. Han, C. Carpenter, G. Pratx, Y. Kuang, L. Xing, "X-ray induced photoacoustic tomography," Proc. SPIE 8581, 85811I (2013).

16. Editorial, "Beyond the diffraction limit," Nature Photonics 3, 361 (2009).

17. A. Harootunian et al., "Superresolution fluorescence near-field scanning optical microscopy," Appl. Phys. Lett. 49(11), 674-676 (1986). 
18. S. W. Hell, J. Wichmann, "Breaking the diffraction resolution limit by stimulated-emission - stimulated-emission-depletion fluorescence microscopy," Opt. Lett. 19(11), 780-782 (1994).

19. M. F. Paige, E. J. Bjerneld, W. E. Moerner, "A comparison of through-the-objective total internal reflection microscopy and epifluorescence microscopy for single-molecule fluorescence imaging," Single Mol. 2(3), 191-201 (2001).

20. M. J. Rust, M. Bates, X. Zhuang, "Sub-diffractionlimit imaging by stochastic optical reconstruction microscopy (STORM)," Nat. Methods. 3(10), 793-795 (2006).

21. E. Betzig et al., "Imaging intracellular fluorescent proteins at nanometer resolution," Science $\mathbf{3 1 3}$ (5793), 1642-1645 (2006).

22. A. Gahlmann, W. E. Moerner, "Exploring bacterial cell biology with single-molecule tracking and superresolution imaging," Nat. Rev. Microbiol. 12(1), 9-22 (2014).

23. L. V. Wang, S. Hu, "Photoacoustic tomography: In vivo imaging from organelles to organs," Science 335(6075), 1458-1462 (2012).

24. H. M. Gu, S. H. Yang, L. Z. Xiang, "Photoacoustic tomography and applications in the medical clinic diagnosis," Prog. Biochem. Biophys. 33(5), 431-437 (2006).

25. L. Z. Xiang et al., "4-D photoacoustic tomography," Sci. Rep. 3, (2013).

26. L. Z. Xiang et al., "Photoacoustic imaging of blood vessels based on modified simultaneous iterative reconstruction technique," Acta Phys. Sin. 56(7), 3911-3916 (2007).

27. L. Z. Xiang et al., "In vivo monitoring of neovascularization in tumour angiogenesis by photoacoustic tomography," Chin. Phys. Lett. 24(3), 751-754 (2007).

28. L. Z. Xiang et al., "Photoacoustic molecular imaging with antibody-functionalized single-walled carbon nanotubes for early diagnosis of tumor," J. Biomed. Opt. 14(2), 021008 (2009).

29. X. J. Yang, L. Z. Xiang, "Photoacoustic imaging of prostate cancer," J. Innov. Opt. Health Sci. 10(4), 1730008 (2017).

30. P. Samant, J. Chen, L. Z. Xiang, "Characterization of the temperature rise in a single cell during photoacoustic tomography at the nanoscale," J. Biomed. Opt. 21(7), 75009 (2016).

31. P. Samant, J. Chen, L. Xiang, "Photoacoustic tomography of unlabelled red blood cell at the nanoscale," Proc. SPIE Nanoimaging and Nanospectroscopy IV, Vol. 9925 (2016).

32. P. Samant, J. Chen, L. Xiang, "Characterization of the temperature rise in a single cell during photoacoustic tomography at the nanoscale," J. Biomed. Opt. 21(7), 075009 (2016).

33. P. Samant, A. Hernandez, S. Conklin, L. Xiang, "Nanoscale photoacoustic tomography (nPAT) for label-free super-resolution 3D imaging of red blood cells," Proc. SPIE Nanoimaging and Nanospectroscopy V, Vol. 10350, p. 103500P (2017). 